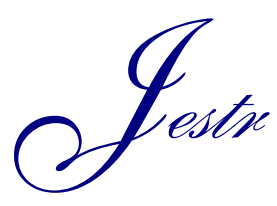

Journal of Engineering Science and Technology Review 5 (1) (2012) 1-6

JOURNAL OF

Engineering Science and

Technology Review

Research Article

www.jestr.org

\title{
Design and performance considerations in stand-alone PV powered telecommunication systems
}

\author{
E. Kaplani *
}

Dept., T.E.I. of Patras, Meg. Alexandrou 1, Patra, Greece.

Received 8 December 2011; Accepted 18 February 2012

\begin{abstract}
With the continuous increase observed in stand-alone photovoltaic (SAPV) systems powering telecommunication stations worldwide, the deeper understanding of factors which significantly affect the short and long term performance of the SAPV becomes a necessity. This paper analyses design and performance considerations and factors that affect the performance of the SAPV such as PV sizing, the effect of shadowing, temperature, and dust, and shows how these can lead to reduction in power output of the SAPV and cause a permanent progressive degradation of the PV cells and the entire PV generator. The need for standardized maintenance procedures and remote monitoring and inspection is highlighted, and a careful design phase is promoted for SAPV systems to remain a preferred power alternative for telecommunication applications.
\end{abstract}

Keywords: SAPV in Telecommunications, shadowing effects, PV ageing, IR image and data analysis.

\section{Introduction}

The use of solar energy to power telecommunication systems has greatly increased during the last decade. To provide coverege in all areas, telecommunication systems in several cases need to be installed in remote areas, usually far away from the electricity grid [1]. In these cases, the integration of stand alone photovoltaic (SAPV) systems to power telecommunication stations provide a clear advantage and a potentially reliable solution. Futhermore, nowadays due to the increase of photovoltaic production, their ease of installation and application, and the worldwide directive for an expansion of clean energy use and energy savings, the application of photovoltaics in telecommunication systems to provide a reliable green energy solution has greatly expanded to systems often placed in close proximity to the grid.

Telecommunication stations have generally low power requirements in the range of $10 \mathrm{~kW}$ [2], with a daily energy consumption in the range of $70-150 \mathrm{kWh}$ depending on technology and demand a relatively constant DC load profile, for transmission functions of about $800 \mathrm{Wh}$ during a day, along with an AC energy consumption, including cooling/heating of the base station, during warm/cold hours of the day, which increases the energy consumption by about $1 / 3$ [3]. Due to their low energy consumption, telecommunication systems is a preferred application for stand alone PV systems. However, due to mass production and installation in many cases the integration of stand alone photovoltaic systems in telecommucation applications, lacks of a carefull design, optimum sizing configuration, effective installation specifications, and most of the times operational monitoring and maintenance processes.

An onsite visit at a SAPV powered telecommunication system for a visual inspection may often reveal design weaknesses or errors and factors leading to substantial power losses, also responsible for a potential decrease in the operational life time of the SAPV system. Such weaknesses may be seen in the sizing of the PV system, when the specific climatic conditions and solar radiation data of the site have not been thoroughly considered, the inclination of the PV generator is not optimum to supply the maximum yield, when dust covers or surrounding objects shadow the PV panels leading to a substantial perfomance degradation and decrease in the operational life time of the system [4].

This paper analyses critical design considerations and performance indicators that need to be taken into account for a SAPV system to provide reliable, uninterrupted and durable service in telecommunication applications and remain a power supply cost-effective solution.Due to the increasing concern about the global warming, renewable energy systems especially wind energy generation have attracted great interests in the recent years. Large wind farms have been installed or planned around the world and the power ratings of the wind turbines are increasing. For many wind farms, wind turbines based on doubly fed induction generator technology are used.

\footnotetext{
* E-mail address: ekaplani@teipat.gr

ISSN: 1791-2377 @ 2012 Kavala Institute of Technology. All rights reserved.
} 


\section{General requirements of a SAPV powered telecommunication system}

A SAPV powered telecommunication system requires PV generator of peak power usually in the range of $2-10 \mathrm{kWp}$ and battery system capacity in the range of 1-6 kAh [5]. The integrated SAPV system needs to provide energy autonomy for the telecommunication system to operate uninteruptedly and meet the constant DC loads for the signal transmission and the DC/AC loads required for the air conditioning facility, telecom and monitoring equipement, the lights and other auxiliaries.

The general requirements of a SAPV system for telecommunication applications are:

- providing energy autonomy, with a reliability level above $99 \%$ for critical loads and at least $95 \%$ for the non-critical [6].

- uninterrupted service

- operation under extreme weather conditions

- low service and maintenance requirements

- lightning protection of devices

- providing constant temperature and low humidity level in the battery room.

- high operational lifetime of system

- alarm functions signalling the central station

- remote monitoring of system

- cost-effectiveness

\section{Design considerations}

For a reliable and cost-effective SAPV system powering a telecommunication station, there are a number of general issues that need to be thoroughly considered:

- solar radiation profile during the days of the year and meteorological conditions at the site

- characteristics of surrounding area and ground

- required level of reliability for operation of the SAPV system. It is important to consider the critical and the non-critical loads, in order to estimate the factor of energy autonomy in the most cost-effective way.

- sizing issues such as: type of PV module, size of PV array, connection of modules, battery capacity and number of battery cells, optimum angle of inclination and orientation of the PV panels, and level of elevation

- use of fixed or sun-tracking system

- ageing effects of the PV panels [7]

- ageing effects of the Batteries

- all load profiles

- total power losses

- protection of the facility

- means of monitoring, remote inspection, and required maintenance

Depending on the decision made on several of the above issues, the system configuration may exhibit a different level of reliability, service requirements, durability and cost-effectiveness. Furthremore, there are several factors that affect the performance of the SAPV system which may be minimized or avoided altogether if a carefull design and consideration of the above is performed.

\section{A. Sizing}

The sizing of the SAPV system needs to be carried out based on knowledge acquired on the climatic conditions of the site and on a thorough analysis of past years solar radiation data on the site in order to provide for both reliability and cost-effectiveness. The latter is an important issue as solar radiation exhibits inherent fluctuations following a Weibull or Extreme Value distribution [8]. It is often observed that SAPV systems are oversized in order to provide for high reliability. This means larger PV arrays and battery system, and of course higher cost for installation and maintenance. A stochastic simulation algorithm was proposed in [8] for the sizing of SAPV systems which, based on a statistical analysis of solar radiation data on a site, determines the minimum installed peak power and battery capacity at different levels of required reliability for critical and non-critical loads, i.e. $99 \%$ and $95 \%$ respectively. According to this method the estimated peak power $\left(\mathrm{P}_{\mathrm{m}}\right)$ and battery capacity $\left(\mathrm{C}_{\mathrm{L}}\right)$ are given by the following equations:

$$
\begin{aligned}
& P_{m}=Q_{L} \cdot F \cdot\left(1+\sqrt{d} \cdot 2 \cdot \sigma_{H} / H_{m}\right) /\left(P S H_{m} \cdot R_{m}\right) \\
& C_{L}=Q_{L} \cdot F^{\prime} \cdot\left(1+\sqrt{d} \cdot 2 \cdot \sigma_{H} / H_{m}\right) /(V \cdot D O D)
\end{aligned}
$$

where $\mathrm{Q}_{\mathrm{L}}$ is the total loads in a day in $\mathrm{Wh} /$ day; $\mathrm{F}$ is a correction factor for loads due to energy dissipation in the entire system, and F' the respective correction factor due to energy dissipation from the battery to the loads; V is the DC transfer voltage; DOD the depth of discharge of the battery; $\mathrm{H}_{\mathrm{m}}$ the mean value of the daily global solar radiation on the horizontal and $\sigma_{\mathrm{H}}$ its standard deviation, for the site and the month on which sizing is based; $\mathrm{PSH}_{\mathrm{m}}$ the mean peak solar hour (h/day) and $\mathrm{R}_{\mathrm{m}}$ a conversion factor for the solar radiation from the horizontal to the inclined PV array (mean value for the specific month the sizing is based on, and the specific site).

According to this stochastic sizing model, $d$ is determined through an iterative algorithm which simulates the performance of the entire SAPV system - PV generator and battery bank - and can be estimated depending on the level of system reliability required. This methodology showed to bring significant reduction in installed peak power and battery capacity compared to the conventional sizing approach based on the number of days of autonomy $[9,10]$. Furthermore, several optimum sizing methodologies or simulation algorithms have been reported based on stochastic processes [11, 12], Neural Networks [13], Hidden Markov Models [14], and the concept of Loss of Load Probability (LLP). Thus, according to the profile of the solar radiation of the site in question, the optimum size of the PV array and battery storage system may be determined to provide energy autonomy at the highest required reliability level.

The type of the PV modules needs to be selected as a balance between module efficiency and cost. Also, the interconnection of cells in the PV module -whether in parallel or in series - and the number of bypass diodes integrated in the PV module, along with the interconnection of modules in the PV array play a very important role. It is very often observed, that shading of cells occurs, which can be detrimental to the performance of the system, evident in the I-V curve shape and the power output, and detrimental to its long-term lifetime, as it enhances factors of PV cell degradation [7]. The performance degradation of the system can be partly minimized with the use of series-parallel configuration [15] and a greater number of bypass diodes to 
protect each cell string.

\section{B. Shadowing}

The climatic characteristics at the site and the infrastructure of the surrounding area of the telecommunication station where the SAPV system is to be installed need to be particularly considered. Due to the environmental conditions in Greece, several SAPV systems installed at telecommunication stations are surrrounded by trees or bushes, as seen in Fig. 1.

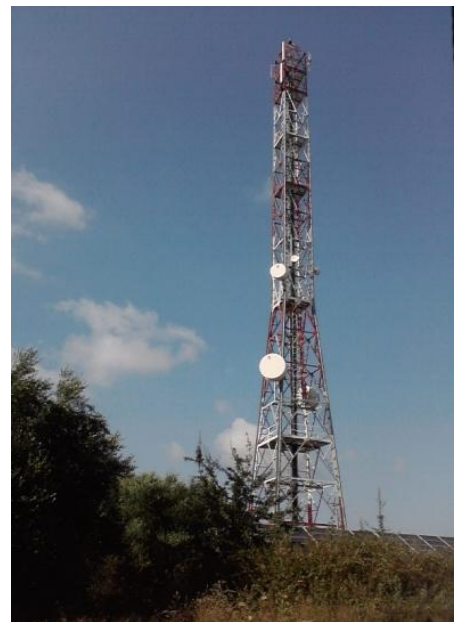

Fig. 1. A PV powered telecommunication system near Pyrgos, Greece.

In these cases, if neglected, may partly shadow the PV array and cause serious performance degradation and, eventually long-term damage to the PV cells and modules.

Shadowing often also occurs from nearby objects, poles, or even the fence installed for protecting the area of the station. In many cases these have not been properly considered or foreseen at design phase, see for instance Fig. 2(a) and (b) of the same PV array taken at diffent times of the year. The big shadow in Fig. 2(b) is caused by a nearby pole and covers $11 \mathrm{PV}$ modules of the array leading to substantial reduction in the PV output. A closer look also reveals a smaller shadow caused by the surrounding fence placed to protect the site, which will become larger at later hours of the day.

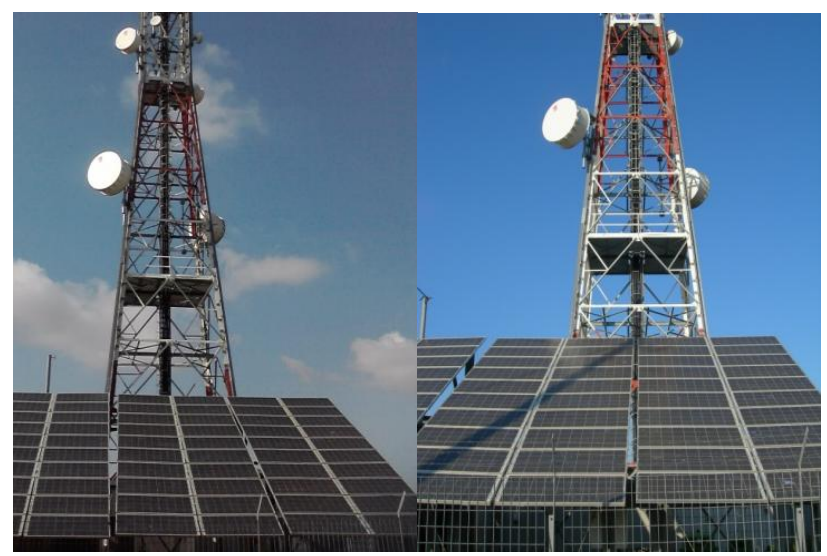

(a)

(b)

Fig. 2. PV generator powering a telecommunication station (a) picture taken in July 2011, no shadow appears, (b) picture taken in December 2011, shadow caused by a near by pole, covers several PV modules.
The effect of shadow caused by fences or poles on the performance of the PV panels may be illustrated through an experiment performed on a BIOENERGY PV panel and the I-V curves obtained before and at the time it was partially shadowed by a pole, see Fig. 3. The PV panel consists of 72 cells connected in series. The shadow crosses the 6 series of the panel, see Fig.4. The I-V curves were obtained using an I-V curve analyser developed in the R.E.S. lab. The large difference in the current output observed in the two I-V curves is due to the different solar irradiation, as before the shadow the solar irradiation was measured at $519 \mathrm{~W} / \mathrm{m}^{2}$ and when the panel was partially shadowed the solar irradiation was $421 \mathrm{~W} / \mathrm{m}^{2}$.

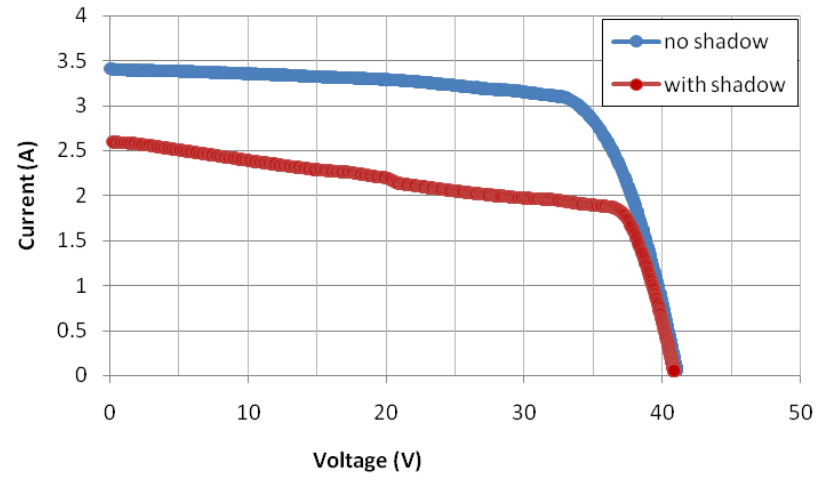

Fig. 3. I-V curve of a PV panel before and at the time it was partially shadowed by a pole.

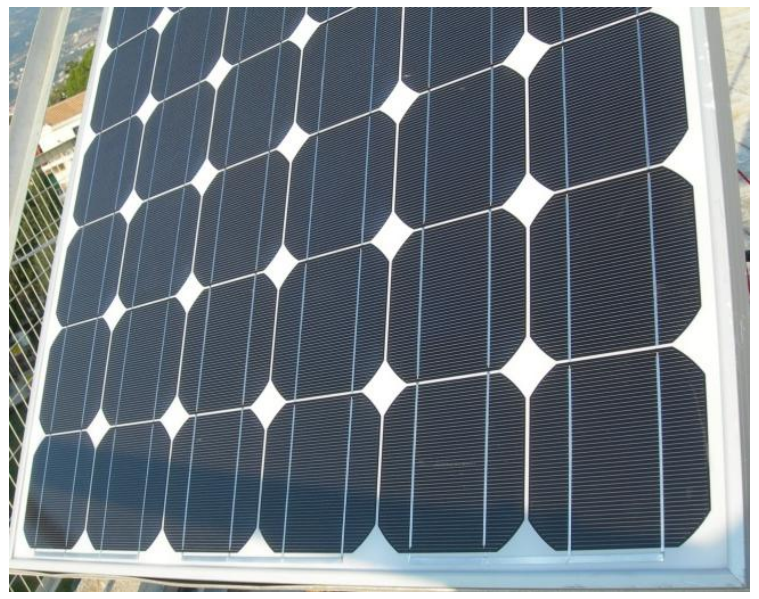

Fig. 4. PV panel partially shadowed by a pole. The thick line of the shadow in the bottom part of the panel crosses all the 6 strings, which are at vertical direction.

The shadow effect in the red I-V curve is realised through the step-wise reduction observed. If the maximum peak power measured is converted to Standard Test Conditions (STC) for comparison, then before the shadow the $\mathrm{P}_{\mathrm{m}}(\mathrm{STC})$ was $203 \mathrm{~W}$, whereas when the panel was partially shadowed the $\mathrm{P}_{\mathrm{m}}(\mathrm{STC})$ was $166 \mathrm{~W}$, a relative reduction of $18 \%$. Furthermore, before the shadow the fill factor (FF) converted to STC was estimated $73.8 \%$, whereas, when the panel was partially shadowed, FF(STC) was estimated equal to $64.6 \%$, a relative reduction of $12.5 \%$ in PV performance.

The degree to which the shadow affects the performance output of a PV generator depends on the surface covered by the shadow, the interconnection of the cells, the way they are connected to the bypass diodes, and the interconnection of 
the panels. These need to be particularly considered in order to decrease a potential shadowing effect. The reduction in performance output can be substantial and especially crucial for telecommunication applications where high reliability and uninterrupted service is a requirement.

\section{Temperature effect}

The effect of partial shadowing is not only seen in short term reduction of power output, but it can also cause long term degradation of the cells and panels. This is due to the internal temperature increase in the cells as a result of partial shadowing, which can permanently damage the cell and degrade the performance of the panel if it appears systematically and remains for a long period of time.

In a further experiment performed via means of an IR camera TROTEC IC080LV to capture the infrared image of the temperature distribution on the surface of a 2 year old panel partially shadowed by a nearby fence, it may be seen how the shadow affects the temperature of the cells (see Fig. 5 (a) and (b)). In Fig. 5(a) the dark thick line is the shadow falling on the 2 corner cells of the panel. It may be observed how the cells which are partially covered by the shadow exhibit a temperature increase by more than $20^{\circ} \mathrm{C}$ compared to the temperature of the neighbouring cells. Fig. 5(b) shows the same panel half hour later when the shadow of the nearby fence has risen and now covers the 2 nd and 3 rd cell in the last row of the panel. The same effect is observed now in the cells covered by the shadow. Note that as the shadow has moved over the top of the corner cell its temperature has droped to the temperature of the neighbouring cells. This experiment was performed in the beginning of November. It was observed that during summer the temperature rises in the vicinity of $90^{\circ} \mathrm{C}$ degrees. This is detrimental to the performance of the cells as it may lead hot spots when the shadow remains for a long time.

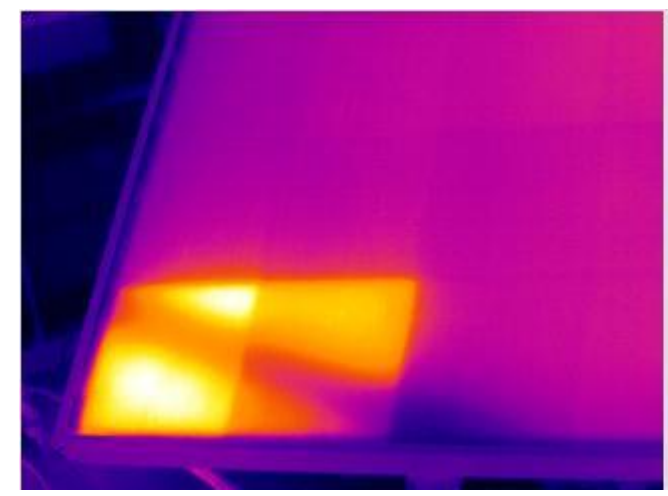

(a)

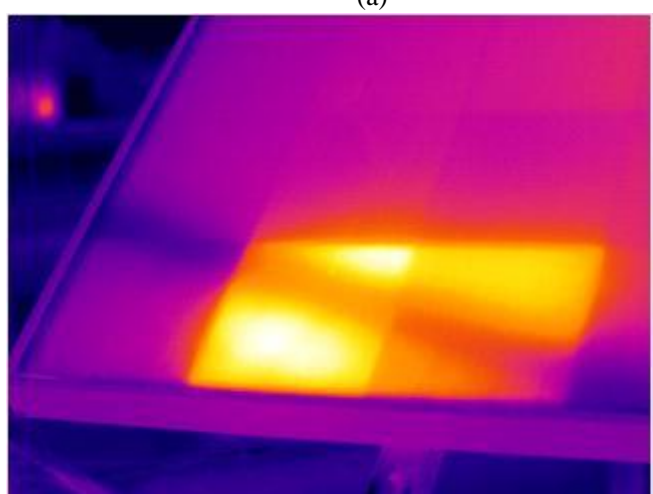

(b)

Fig. 5. Infrared image of a 2.5 year old PV panel when partially shadowed by a nearby fence, RES Lab, T.E.I. of Patras.
The shadowing effect may also be observed on cells partially covered by bird pits. This phenomenon is very often observed on the cells of PV generators at telecommunication stations in Greece, since, as earlier mentioned, telecommunication stations are often installed in the countryside whereby trees and bushes are in the surrounding area attracting birds and insects. Fig. 6 shows the thermographic image of several cells of an 18 year old SIEMENS M55 PV panel. The hot cell which appears on the image is a cell which was covered with mortar for an entire season, which led to severe ageing of the cell with permanent degradation. The cell just above was partially covered by a bird pit when the thermography was taken. This shows a temperature increase at that spot of the cell by about $10^{\circ} \mathrm{C}$ compared to the temperature of the entire cell. Thus, it is evident that when a shadow repeatedly occurs for a prolonged period it will eventually lead to the degradation of the cells and the panel.

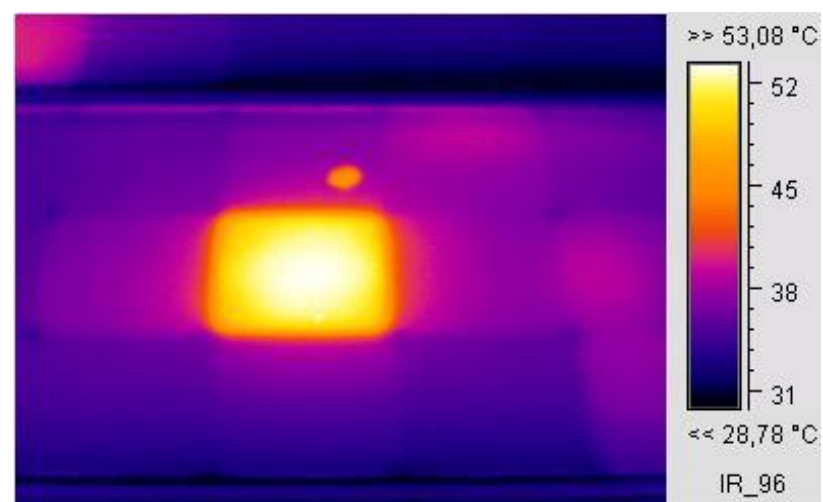

Fig. 6. Infrared image of several cells of an aged PV panel, showing a permanently degraded cell exhibiting increased temperature and the temperature increase due to a bird pit on the cell just above; RES Lab, T.E.I. of Patras.

Furthermore, the temperature effect alone, due to increased ambient temperature leads to a power drop in the output of a panel compared to the nominal power measured at STC. Based on the energy balance equation, the temperature of a lighted PV cell may be described by the following equation [16]:

$T_{c}=T_{a}+\lambda \cdot \mathrm{I}_{\mathrm{T}}$

where $T_{c}$ is the temperature of the cell, $T_{a}$ is the ambient temperature, and IT is the solar radiation on the inclined PV panel. $\lambda$ is a coefficient measured in $\mathrm{m}^{2}{ }^{\circ} \mathrm{C} / \mathrm{W}$, which depends on the inclination of the PV panel and the wind velocity. A value of $\lambda=0.03$ was estimated as in [16].

Futhermore, the final power drop $\delta \mathrm{Pm} / \mathrm{Pm}$, may be described by the following equation:

$$
\frac{\delta P_{m}}{P_{m}} \%=-0,4 \% /{ }^{\circ} \mathrm{C} \cdot\left(T_{C}-25^{\circ} \mathrm{C}\right)
$$

Thus, in the Summer for an ambient temperature of $30^{\circ} \mathrm{C}$ and a solar irradiation of $103 \mathrm{~W} / \mathrm{m}^{2}$, the final power drop would be estimated at $14 \%$. Therefore, the reduction in power output due to the temperature effect alone is 
also an important consideration for the SAPV system to power telecommunication stations at countries with hot climates.

\section{Dust}

The dust that accumulates on PV generators when left uncleaned for a long period of time, is also a cause of light shadowing effect and reduction in power output [17]. In African, Middle East, or even South European countries, where dusty winds are very prominent, a heavy dust gets stuck on the PV panels and heavily affects their performance. Fig. 7 shows a SAPV system powering a telecommunication station in the middle of the desert south to Kairo. The dust remaining on the PV panels leads to a substantial decrease in its power output and this should be thoroughly considered at design phase. The use of sun tracking systems in telecommunication application has been observed to minimize the dust on the panels and as a result, apart from the other benefits of the sun tracking systems, increase the performance of the SAPV.

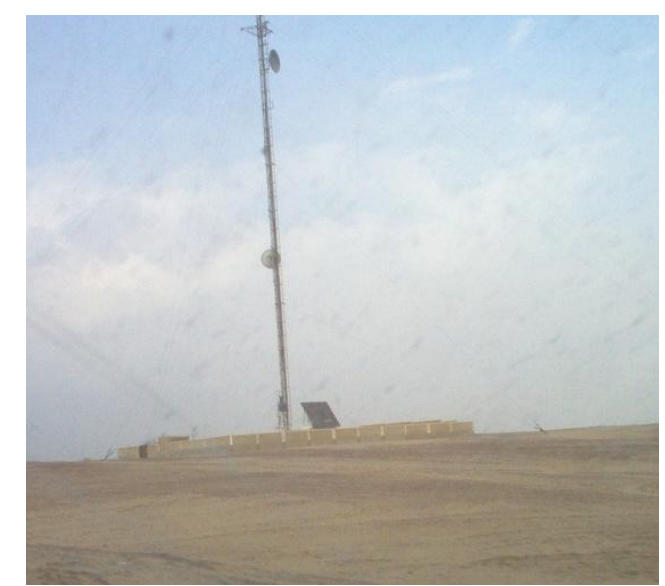

Fig. 7. SAPV system powering a telecommunications station in the middle of the desert outside Kairo, Egypt.

The accumulated dust on the PV generator of the telecommunication station of Fig. 2, may be observed in the zoomed in picture shown in Fig. 8.

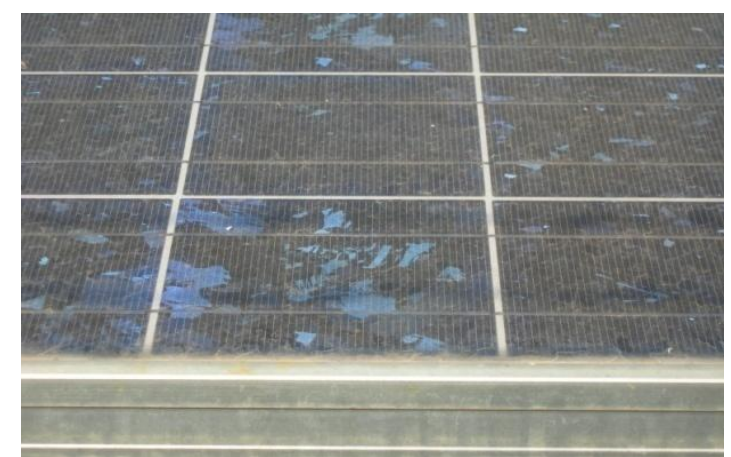

Fig. 8. A close up look showing the amount of dirt and dust covering the PV cells of the SAPV system powering the telecommunication station shown in Fig. 2.

To investigate the effect of dust in a further experiment, the electrical characteristics of a 13 year old
SIEMENS SM55 PV panel were obtained using an I-V curve analyser developed in the R.E.S. lab. The experiment was carried out when the panel was naturally covered in finely dispersed dust operating in field conditions and after it was cleaned. The solar radiation on the dusty panel was $697.7 \mathrm{~W} / \mathrm{m}^{2}$ with panel temperature $43.4^{\circ} \mathrm{C}$ and after it was cleaned $629.2 \mathrm{~W} / \mathrm{m}^{2}$ and panel temperature was $38.6^{\circ} \mathrm{C}$. As the solar radiation and temperature were different during the experiments, the electrical parameters were converted to STC for comparison reasons and are presented in Table 1.

Table 1. Electrical parameters of PV panel at STC when the panel was naturally covered in dust and after it was cleaned.

\begin{tabular}{lcccc}
\hline & Pa (STC) in W Voc(STC) in V & Isc(STC) in A & FF(STC) $\%$ \\
\hline dusty panel & 50.37 & 19.83 & 3.74 & 68,0 \\
\hline clean panel & 53.01 & 19.74 & 3.91 & 68.7 \\
\hline
\end{tabular}

The relative decrease in the power output caused by the dust was $5.2 \%$ while the FF was reduced by $1 \%$. Although the increase in performance is not dramatic after the panel was cleaned, the improvement is obvious. The effect would be more obvious in a PV generator consisting of several PV panels, or in SAPV systems installed in areas were the dust is heavier and the panels have not been cleaned for a long period of time. Thus, the importance of maintaining the PV panels cleaned, free of dust and dirt may be realised.

\section{Discussion}

The factors analysed in the previous section were shown to cause both significant reduction in power output but also a permanent effect leading to PV cell and panel degradation reducing the opeartional durability of the system.

These factors need to be thoroughly considered at design, installation and maintainance phase, in order to secure performance reliability and cost-effectiveness, foreseeing and minimizing the effects which lead to performance degradation, and, furthermore, prolonging operational lifetime of the SAPV system. These are especially important if SAPV systems are used to serve telecommunication applications, which require high performance reliability, uninterrupted service and durability.

Monitoring the SAPV system is an important task and does not necessarily imply regular visits, service and maintenance costs, but can be implemented remotely by several means. External cameras capturing the image of the PV generator, telecommunications station and surrounding area in order to detect any shadowing effects or malfunction may be used; data from temperature sensors, humidity sensors, battery sensors, etc., as well as the I-V curves from series of panels need to be recorded at regular intervals and automatically analysed for detection of any unreasonable power drop. An alarm function at different levels of severity can signal the maintenance department in order to initiate the appropriate action. Different maintainance procedures 
need to be applied according to design.

A careful design in terms of the selection of appropriate PV system, whether sun-tracking system rotating on one or two axis, or fixed system, otpimum PV panel inclination, PV cell technology, panel characteristics, cell and panel interconnections, bypass and blocking diodes used, optimum sizing of PV and batteries for the solar radiation data and climatic conditions at the site, also withstanding temperature and ageing effects; along with an adequate monitoring and maintenance standardized process will gurantee a highly reliable SAPV system performance.

\section{Conclusions}

The research work presented in this paper has examined and analysed several factors, which significantly affect the performance of SAPV systems powering telecommunication applications. As SAPV powered telecommunication stations nowadays are becoming a norm, it is often observed even via visual inspections at the sites, that the sizing of the SAPV is not optimum; that shadows caused by nearby objects, poles or protection fence may partially cover the PV generator; or that the PV panels are heavily covered in dust, dirt or bird pits and left uncleaned for a prolonged period of time. The effect of these factors has been analysed and shown to cause significant short and long term performance degradation both in terms of the power output produced but also with respect to the durability of the PV system as the effects of shadowing and temperature lead to a permanent and progressive degradation of the cells, and, furthermore, the degradation of the entire PV system.

Although SAPV systems are often promoted as a reliable and cost-effective solution for powering telecommunication stations in remote areas whereby minimal maintainance is required, in fact it is shown in this work that monitoring the SAPV system both in terms of performance and external effects is crucial and could guarantee preserving both a highly reliable performance of the system and its operational durability. As discussed in the previous section, adequate monitoring can be carried out dynamically and maintenance actions can be initiated through alarm functions after a possible problem or error has been automatically detected. Nevertheless, the importance of implementing adequate standardized maintenance procedures should be stressed, in order to preserve the preeminence, reliability, durability and costeffectiveness of SAPV systems powering telecommunication stations which may be seen as a worthwhile investment and necessity.

\section{References}

1. M. Soursos, C. Protogeropoulos, P. Suuronen. "Stand-alone photovoltaic systems for telecommunication stations in Greece. 2nd World Conf. and Exhibition on Photovoltaic Solar Energy Conversion, Vienna, Austria, 1998.

2. P. Nema, R.K. Nema, S. Rangnekar. "PV-solar/wind hybrid energy system for GSM/CDMA type mobile telephony base station. International Journal of Energy and Environment, 1(2):359-66, 2010.

3. C. Lubritto. "Telecommunication Power System: energy saving, renewable sources and environmental monitoring. Trends in Telecommunications Technologies, Ed. C.J. Bouras, Intech 2010.

4. E.L. Meyer, E.Ernest Van Dyk. "Assessing the reliability and degradation of photovoltaic module performance parameters. IEEE Transactions on Reliability, 53(1):83-92, 2004.

5. S. Ghozzi, K. Mahkamov. "Case Study: Modelling and sizing stand-alone PV systems for powering mobile phone stations in Libya. World Renewable Energy Congress, Linkoeping, Sweden, 8-13 May, 2011.

6. E. Kaplani, S. Kaplanis. "A stochastic simulation model for reliable PV system sizing providing for solar radiation fluctuations". Proc. of 3rd Int. Conf. on Applied Energy (ICAE), Perugia, Italy, 16-18 May, 2011.

7. S. Kaplanis, E. Kaplani. "Energy performance and degradation over 20 years performance of BP c-Si PV modules". Simulation Modelling Practice and Theory 2011; 19: 1201-11.

8. E. Kaplani, S. Kaplanis. "A stochastic simulation model for reliable PV system sizing providing for solar radiation fluctuations". To appear in Applied Energy, doi 10.1016/j.apenergy.2011.12.016.

9. RETScreen International, Photovoltaic Project Analysis, web site: www.retscreen.net

10. Messenger R, Ventre J. Photovoltaic Systems Engineering. USA: CRC Press; 2000.

11. Markvart T, Fragaki A, Ross JN. PV system sizing using observed time series of solar radiation. Solar Energy, 80: 46-50, 2006.

12. Kaplanis S, Kaplani E. Stochastic prediction of hourly global solar radiation for Patra, Greece. Applied Energy, 87:3748-58, 2010.

13. Kalogirou SA. Artificial neural networks in renewable energy systems applications: a review. Renewable and Sustainable Energy Reviews, 5: 373-401, 2001.

14. Hocaoglu FO. Stochastic approach for daily solar radiation modelling. Solar Energy 85: 278-87, 2011.

15. P. Spirito and V. Albergamo. "Reverse bias power dissipation of shadowed or faulty cells in different array configurations". PV Solar Energy Conference. Proc. of 4th Int. Conf., Stresa, Italy, May 1982.

16. S. Kaplanis, E. Kaplani, P. Wolf. "On the effect of the PV array inclination to its performance and efficiency". Proc. of World Renewable Energy Congress (WRECX), Glasgow, UK, 2008.

17. J.K. Kaldellis, M. Kapsali. "Simulating the dust effect on the energy performance of photovoltaic generators based on experimental measurements". Energy 36:5154-61, 2011. 